\title{
Valoración por el consumidor de las características hedónicas, nutritivas y saludables del amaranto
}

\section{Consumer assessment of hedonic, nutritional and healthy characteristics of the amaranth}

\author{
Carlos Aragón Gutiérrez ${ }^{\mathrm{a} \star}$, Benito Ramírez Valverde ${ }^{\mathrm{b} \star}$, María José Montero Simóc ${ }^{c \star}$, \\ Rafael Ângel Araque Padilla ${ }^{\mathrm{d} \star \star}$, José Javier Pérez Barea ${ }^{\mathrm{\star} \star \star}$
}

\section{RESUMEN}

La finalidad del estudio es conocer en qué medida los consumidores valoran las propiedades sensoriales, hedónicas, nutritivas y saludables de alimentos con alto valor nutritivo, como es el caso del amaranto. El estudio se fundamentó en la teoría del Valor Percibido por el Consumidor (VPC) y para obtener la información se utilizó un cuestionario con preguntas cerradas, aplicado a una muestra de 341 consumidores. Para el análisis estadístico se utilizó un modelo de ecuaciones estructurales. Los resultados obtenidos indican que el precio y la calidad, son los componentes más importantes en la conformación del VPC en el amaranto, en tanto que sus características hedónicas están muy cerca de equipararse con esos dos componentes. La nutrición es la que menos contribuyó a la creación del VPC, y en cuanto a sus propiedades benéficas para la salud, éstas son desconocidas por la mayoría de los encuestados.

\begin{abstract}
The aim of the study is to know the extent to which consumers value the sensory, hedonic, nutritional and healthy properties of foods with high nutritional value such as amaranth. The study was based on the theory of Consumer Perceived Value (CPV). To obtain information, a questionnaire with closed questions was applied to a sample of 341 consumers. For statistical analysis, a structural equation model was used. The results obtained indicate that price and quality are the most important components in CPV regarding amaranth, while its hedonic characteristics are very closely equated with these two components. Nutrition is the characteristic that contributed least to the creation of the CPV, and in terms of its beneficial properties for health, these are unknown by most of the respondents.
\end{abstract}

^Colegio de Postgraduados,

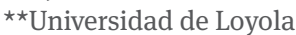

Recibido: 16 de octubre de 2017; aceptado: $28 \mathrm{de}$ febrero de 2018

\section{G:}

Palabras clave: nutrición, salud, obesidad, valor percibido por el consumidor.

G

Keywords: nutrition, health, obesity, consumer perceived value.

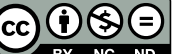

Se autoriza la reproducción total o parcial de los textos aquí publicados siempre y cuando se cite la fuente completa y la dirección electrónica de la publicación. CC-BY-NC-ND 


\section{INTRODUCCIÓN}

Los alimentos permiten satisfacer una necesidad biológica ineludible porque de su plena satisfacción depende la conservación de la vida. Además, son estímulo placentero para los órganos de los sentidos; instrumento eficaz de comunicación y de vinculación social; medio básico de expresión cultural y estética; y elemento medular de ritos, ceremonias, celebraciones y del sentido de identidad de una sociedad. Por lo tanto, la dieta, es decir el conjunto de alimentos ingeridos durante el día, será correcta si satisface los requerimientos biológicos, psicoemocionales y socioculturales (Kaufer, Pérez y Arroyo, 2015).

En cuanto a lo biológico, la dieta debe ser equilibrada, es decir, aportar todos y cada uno de los nutrimentos necesarios, en las cantidades suficientes pero no excesivas, atendiendo al estado fisiológico particular de cada individuo. La escasez o exceso de un nutrimento afectará la salud de la persona. Por ejemplo, una de las principales afecciones relacionadas con una excesiva ingestión de grasas y carbohidratos es la obesidad (Astiasarán y Martínez, 2000).

Respecto a lo psicoemocional, debe ser agradable a los sentidos y variada, requiriendo un mínimo esfuerzo para obtenerla y consumirla. En cuanto a lo sociocultural, debe ser compatible con las tradiciones, hábitos, preferencias e ingreso económico de la comunidad a la que se pertenece (Kaufer et al., 2015).

Aunado a lo anterior, se ha identificado a un conjunto de alimentos que además de aportar nutrimentos, puede proporcionar beneficios especiales para la salud, previniendo o contribuyendo a curar enfermedades. En contraparte, muchas de las enfermedades que en la actualidad causan mayor morbilidad y mortalidad, tienen un importante componente alimentario. Esto se debe al desvío que tiene la dieta apropiada para una persona a causa de múltiples factores. Kaufer et al. (2015) mencionan algunos factores como la disponibilidad local de alimentos y el acceso de la población a los alimentos disponibles debido al poder adquisitivo.

México ocupa, el primer lugar mundial en obesidad infantil, y el segundo en obesidad en adultos. La obesidad y el sobrepeso afectan a $67 \%$ de los hombres adultos y $72 \%$ de las mujeres adultas. A largo plazo, la obesidad favorece la aparición de enfermedades tales como dia-

DOI: $10.22201 /$ enesl. 20078064e.2018.16.62956 betes, la cual es el mayor problema al que se enfrenta el Sistema Nacional de Salud, pues es la principal causa de muerte en adultos, la primera causa de demanda de atención médica y la enfermedad que consume el mayor porcentaje de gastos en las instituciones públicas (United Nations International Children's Emergency Fund [UNICEF], s.f.).

Una de las principales causas de lo anterior radica en que gran parte de la población cambió sus alimentos básicos -sopas, tortillas, frijoles, verduras y un poco de carne o leche-por alimentos "chatarra". El cambio se debió a que estos últimos se hicieron disponibles en gran cantidad, en empaques pequeños de fácil conservación, no requieren cocinarse, gustan y llenan el estómago debido a la gran cantidad de azúcares y grasas que contienen, siendo de muy mala calidad porque aportan calorías con muy pocos nutrimentos, fibras y fitoquímicos, además de contener elevadas cantidades de sodio (Chávez et al., 2013), con bajo valor nutritivo por ser muy refinados y sometidos a tratamientos térmicos. A pesar de ello, sus agradables aromas y sabor favorecen su consumo (Astiasarán y Martínez, 200o). También juega un papel muy importante el entorno alimenticio, es decir, el número de puntos de venta de comida rápida, la variedad de comida altamente procesada y la publicidad de los alimentos (Castillo, 2013).

Ante el gran problema nacional que representa la dieta de gran parte de la población caracterizada por exceso de grasas, azúcares y sodio, así como escasez de otros nutrimentos, es apremiante impulsar, en un marco de dieta equilibrada, el consumo de alimentos que aporten nutrimentos en forma sobresaliente, excepto grasas saturadas, azúcares y sodio, privilegiando aquellos que posean cualidades benéficas para la salud. Un ejemplo, puede ser el caso del amaranto, que es un excelente alimento debido a su alto valor nutritivo.

El amaranto (Amaranthus hypochondriacus), planta que pertenece a la familia Amaranthaceae y al género Amarhantus, a nivel mundial tiene su centro de origen y diversidad más importante en México donde ya se cultivaba 5,200 años A. C. Desde mucho antes de la llegada de los españoles, se plantaba extensamente y en el momento de su arribo era uno de los cultivos más importantes en el área de Mesoamérica y aún más al norte. Entre los

Entreciencias 6(16): 3-16. Abr. - Jul. 2018 
Aztecas y sus vecinos el grano tuvo, además, importancia religiosa (Espitia et al., 2010). Esta planta era parte del sustento habitual de la población; sin embargo, fue reemplazado casi en su totalidad por otros cultivos a partir de la época de colonización española (Morán et al., 2012).

Actualmente el amaranto es por su resistencia a sequía una excelente alternativa agronómica para la siembra de cereales en regiones que dependen del temporal. En México el amaranto se cultiva en condiciones de minifundio bajo el régimen de temporal por productores que en su mayoría utilizan herramientas manuales y de tracción animal, con baja o nulo uso de fertilizantes químicos, abonando sus terrenos de cultivo con estiércol de diversos animales, así como de otras fuentes de materia orgánica, favoreciendo la conservación de los recursos naturales y del medio ambiente (Sánchez et al., 2015).

El amaranto cosechado se puede almacenar por un periodo mayor al del maíz sin alterar su calidad. Para su consumo se requiere un proceso de transformación que consiste en someter el grano de amaranto a altas temperaturas $\left(120^{\circ} \mathrm{C}\right)$ para que se infle; ya reventado, se enfría y se criba para separar el grano que no se expandió. El grano reventado se vende como cereal o se utiliza como insumo de otros productos alimenticios.

La comercialización del amaranto es uno de los aspectos más vulnerables de la cadena, particularmente para los productores, puesto que no existen vínculos horizontales entre ellos y no se identifica una cultura de asociación, lo cual debilita la capacidad de negociación de 80\% de los productores que vende el amaranto sin procesar a intermediarios, quienes deciden el precio a inicios de la temporada de cosecha y lo incrementan o disminuyen dependiendo de la oferta y la demanda. El mayor riesgo en la cadena está del lado de los productores, pues la producción depende de las condiciones climáticas; no obstante, la utilidad se incrementa conforme se avanza en los eslabones de la cadena (Ayala et al., 2016).

En años recientes ha surgido un gran interés en el consumo de este grano tanto a nivel nacional como internacional dadas sus propiedades nutritivas. En México se ha consumido tradicionalmente en forma de "alegría" que es una barra elaborada con amaranto mezclada con miel, azúcar o piloncillo y algunos otros ingredientes, cubierta con una sencilla envoltura de celofán que se vende en pequeños puntos de venta (Ayala et al., 2012). En algunos supermercados se ha iniciado la venta de "alegría”, que ahora la denominan mini snacks, con una envoltura llamativa, en una caja de 30 barras, también muy atractiva; el precio de una de estas barras es el triple del que tiene la "alegría" con envoltura tradicional. También se consume en barras energéticas, granola, pan, galletas, tamales, atole, frituras, entre otros productos.

El amaranto tiene un alto valor nutritivo, siendo éste mayor, en términos generales, al que tienen el maíz, trigo y arroz, quienes proveen más de la mitad del aporte energético de la dieta humana actual. Además de poseer un mayor aporte energético que los tres cereales mencionados, también aporta más proteína con un mejor balance de aminoácidos esenciales, así como mayor cantidad de calcio, hierro, magnesio, fósforo y potasio. Otra ventaja es tener una digestibilidad de 93\% (Escobedo, Ayala y Campos, 2012). Según la Organización de las Naciones Unidas para la Agricultura y la Alimentación (FAO, por sus siglas en inglés) y la Organización Mundial de la Salud (oms), sobre un valor proteico ideal de 100, el amaranto posee 75 , la leche vacuna 72 , la soya 68 , el trigo 60 y el maíz 44 (Secretaría de Agricultura, Ganadería, Desarrollo Rural, Pesca y Alimentación [Sagarpa], 2016a).

El amaranto se ha consumido por poblaciones rurales, generalmente originarias, donde, de acuerdo con las condiciones de nuestro país, la mayoría de la población se encuentra en condiciones de pobreza. En esta situación destacan los problemas nutricionales y de salud, así como el reducido acceso a los servicios médicos, por lo que el consumo de amaranto es importante debido a sus propiedades benéficas para la salud. El amaranto contribuye a disminuir la presión arterial, el colesterol y la glucosa en la sangre, posee propiedades antioxidantes y cáncer-preventivas. Al no contener gluten es adecuado para pacientes con enfermedad celiaca (Huerta, Maldonado y Barba, 2012).

Una característica de índole socioeconómica que tiene el amaranto es el bajo costo de productos de alto valor nutritivo elaborados con este grano, quedando al acceso de la población (Escobedo et al., 2012), lo cual es relevante en México debido a que en el año 2014, se encontraban en situación de pobreza 55 millones de mexicanos (es decir, 46\% del total de habitantes), y $23.4 \%$ de la población presenta carencia por acceso a la alimentación (Consejo Nacional de Evaluación de la Política de Desarrollo Social [Coneval], 2015).

Pacheco, Mota y Luna (2017) en un estudio en dos mu- 
nicipios de alta y muy alta marginación en el estado de Oaxaca realizado con niños menores de 5 años, encontró que $1.8 \%$ de los niños tenían problemas de desnutrición, y $5.4 \%$ presentaba problemas de obesidad. En otro estudio con mujeres rurales de 7 estados de la república, se encontró un fuerte problema de malnutrición al obtener que $9.6 \%$ de las mujeres estaban delgadas, pero $29.4 \%$ presentaba sobrepeso y $28 \%$ obesidad (Pérez y Romero, 2018). La desnutrición y la obesidad se encuentran presentes en la población en condiciones de pobreza.

El sustento teórico del presente artículo se encuentra en la teoría económica, particularmente en el ámbito del consumo, puesto que se pretende identificar factores que contribuyan a aumentar la población que consuma "alegría" o la cantidad consumida de ese producto entre las personas que ya lo hacen. Tomando en cuenta las condiciones de la población en pobreza, que es la parte de la sociedad prioritaria en este trabajo, se definió como un principio de formulación, el no requerir, para elevar el consumo, un cambio en la renta de los consumidores o en los precios de la "alegría”. Krugman y Wells (2006) señalan que el cambio en los gustos y preferencias es uno de los principales factores que pueden aumentar la cantidad demandada para cualquier nivel de precios (desplazamiento de la curva de demanda). Entre los elementos que pueden modificar gustos y preferencias del consumidor se encuentran el cambio en la calidad, moda, creencias, y cultura.

Por otra parte, es necesario conocer si la "alegría” es un bien normal o inferior, y en este trabajo se busca obtener indicios acerca de la naturaleza de este bien. Nicholson (2008), señala que dado que la utilidad se refiere a la satisfacción general, es evidente que diversos factores afectarán este indicador. La utilidad de una persona no solo depende de los bienes materiales que consume, sino también de sus actitudes psicológicas, de las presiones de su grupo social, de sus experiencias personales y del entorno cultural en general. Los economistas tienen un interés general por analizar estas influencias, pero normalmente necesitan acotar su enfoque. Por tanto, un principio común que se utiliza, es el del ceteris paribus, que consiste en centrar la atención exclusivamente a las elecciones entre opciones cuantificables, manteniendo constantes todos los demás factores que afectan el comportamiento del consumidor .

Por lo expuesto, el objeto de estudio del presente ar- tículo es la "alegría” y el objetivo de esta investigación es identificar y evaluar las dimensiones que conforman el VPC en el consumo de "alegría” y la influencia de esa valoración en la disposición de recompra. El conocimiento a obtener podría ser un elemento fundamental en la formulación de estrategias orientadas a potenciar el consumo de aquellos alimentos, originarios de la región en la que habite el consumidor, que sobresalgan principalmente en el ámbito nutricional, y de esa manera contribuir a solucionar la problemática que representa la obesidad en México, particularmente para la población en condiciones de pobreza.

La tarea de precisar la valoración que los consumidores hacen de cada una de las características de la "alegría”, tiene como fundamento teórico complementario el concepto del Valor Percibido por el Consumidor (VPC), reportado en la literatura de Mercadotecnia como de alta aceptación para conocer la apreciación que los consumidores hacen de los atributos de un producto o servicio y su influencia en la decisión que toman las personas de comprarlo y continuar o no consumiéndolo. Este conocimiento es fundamental para crear productos con mayor valor para el consumidor y así logar que los prefieran sobre otros productos o servicios.

Se define al VPC como la diferencia entre los beneficios derivados de un producto y los costos asociados a su compra que perciben las personas antes o después de la adquisición y de su uso o consumo. En cuanto a los beneficios derivados de un producto, la tipología formulada por Holbrook (1999), la cual está sustentada en la axiología y en la teoría económica, se considera como una de las propuestas más completas de fuentes potenciales de valor o beneficios (Sánchez, Iniesta y Holbrook, 2009). Esta tipología se conforma de las siguientes ocho dimensiones de valor: 1) excelencia o calidad; 2) eficiencia; 3) estatus; 4) estima; 5) hedonismo; 6) estética; 7) ética, y 8) espiritualidad. Con respecto a los costos asociados, las principales dimensiones propuestas son las siguientes: precio (Petrick, 2002), tiempo (Wenben, 1995), esfuerzo físico y mental (Berry, Seiders y Grewal, 2002), tensión emocional (Parasuraman y Grewal, 2000), dinero adicional al precio del producto (Grewal, Monroe y Krishnan, 1998), riesgos financiero, de desempeño y de privacidad (Chen y Dubinsky, 2003).

Hay que tener presente que las tipologías propuestas pueden enriquecerse con dimensiones adicionales y 
cualquiera o todos los tipos de beneficios y costos pueden ocurrir, y frecuentemente ocurren simultáneamente en diferente grado dentro de una experiencia de consumo (Smith, 1999). Además, la relevancia y el uso de cada dimensión de beneficio o costo dependen de la categoría del producto, del propio cliente, así como del contexto en que se dé la valoración del producto (Sweeney, Soutar y Johnson, 1999). Por ello, es necesario realizar estudios que contribuyan a identificar y validar, en cada categoría de productos, el número y naturaleza de las dimensiones -beneficios y costos- que conforman el VPC y escalas fiables para medir el peso de cada una de ellas (Gallarza, Gil-Saura y Holbrook, 2011).

Para definir las dimensiones de beneficios y costos a estudiar en la "alegría”, se analizaron trabajos en los que se investiga la valoración de los atributos de productos alimenticios. De inicio, se incluyeron las dimensiones calidad y precio por ser de las más relevantes en la mayoría de los estudios sobre diversos productos, como es el caso de los trabajos sobre alimentos realizados por Lehtola et al. (2008) y Jezewska y Krolak (2015) en donde se analiza la calidad, y Richards, Patterson y Hamilton (2007) y Ayyaz, Badar y Ghafoor (2011) el precio, junto con otras dimensiones. También se incluyó el valor hedónico pues se ha identificado que juega un papel importante en la ingesta de alimentos, tal como se señala en los estudios de Chaput et al. (2011) y Nasir y Karakaya (2014). Por supuesto, se seleccionó la dimensión nutrición por ser la función central de los alimentos, y así es considerada en estudios como los realizados por Çağlarırmak y Zeki (2013) y Beeler et al. (2012). La dimensión salud fue incluida en el estudio debido a la tendencia creciente que se está presentando en algunos sectores de la sociedad de diversos países por consumir alimentos saludables a causa de una serie de factores entre los que destacan el aumento de los ingresos familiares y las crecientes preocupaciones sobre la salud debido al cada vez mayor número de trastornos metabólicos como la obesidad, problemas del corazón, diabetes, entre otras, como lo señalan Dutta y Singh (2013). Una acción complementaria fue entrevistar a 6 personas con más de 3 años consumiendo "alegría", quienes ratificaron cuatro dimensiones señaladas en la literatura revisada, excepto la dimensión de salud, es importante mencionar que los entrevistados mencionaron desconocer que este alimento tuviese efectos benéficos en la salud del consumidor.
Para esta investigación se definieron las siguientes hipótesis:

- Hipótesis 1: la calidad de la "alegría" contribuye a aumentar el valor que el consumidor percibe en este alimento.

- Hipótesis 2: las características hedónicas de la "alegría” contribuyen a aumentar el valor que el consumidor percibe en este alimento.

- Hipótesis 3: las propiedades nutritivas de la "alegría” contribuyen a aumentar el valor que el consumidor percibe en este alimento.

- Hipótesis 4: los atributos saludables de la "alegría” contribuyen a aumentar el valor que el consumidor percibe en este alimento -se decidió incluir esta hipótesis con el propósito de tener mayores evidencias que corroboraran el desconocimiento de los beneficios a la salud que tiene este producto.

- Hipótesis 5: el precio de la "alegría” contribuye a disminuir el valor que el consumidor percibe en este alimento.

- Hipótesis 6: el valor que el consumidor percibe en la "alegría" influye positivamente en su disposición de recompra.

\section{Metodología}

El contrastaste de las hipótesis del presente estudio requirió llevarse a cabo con personas que hubiesen consumido "alegría", por lo que la población objetivo del presente trabajo se conformó por los consumidores de "alegría" que acudieron a la Feria del Amaranto en Tochimilco, Puebla, llevada a cabo en noviembre de 2015, en donde se expusieron, para degustación y venta, "alegría” $\mathrm{y}$ otros productos elaborados con amaranto.

Tochimilco es un municipio que se distingue por ser el principal productor de amaranto en México: en el año 2013 produjo $24 \%$ del total nacional, -en el estado de Puebla se produjo $46 \%$ del total nacional- (Sagarpa, 2013). Actualmente el cultivo y producción de amaranto en México ha tenido un repunte: en el año de 1986, la superficie cultivada era de 425 hectáreas y la producción fue de 394 toneladas; en 1996 aumentó a 1,372 hectáreas y 1,679 toneladas; en 2006 se amplió a 2,138 hectáreas y 3,302 toneladas; en 2016 fueron 4,545 hectáreas y 6,052 toneladas (Sagarpa, 2016b). 
Para obtener los datos que permitieran contrastar las hipótesis se utilizó el método de la encuesta, y como instrumento se empleó un cuestionario con preguntas cerradas (en el Anexo 1 se presenta éste), el cual fue elaborado a partir de estudios previos sobre alimentos. Al no haber identificado en la literatura revisada indicadores de medición específicos a las dimensiones de valor en el consumo de "alegría”, se recurrió a seleccionar aquellos utilizados en estudios sobre otros alimentos y confirmar su pertinencia para el trabajo de investigación mediante su contrastación con entrevistas en profundidad a 6 personas que tenían más de 3 años consumiendo "alegría" en el momento de la interacción con ellos. La información proporcionada por estos informantes permitió una mayor comprensión sobre la problemática del amaranto y resultó importante para el diseño del cuestionario. Se efectuó una prueba piloto del cuestionario mediante su administración a 14 personas que acudieron a comprar "alegría” a un punto de venta, confirmando la pertinencia de incluir, en la escala de medición de los indicadores de la dimensión salud, la opción "no sé" para aquellos casos en que no se pueda asignar una calificación a los indicadores por no tener información acerca de ellos.

La encuesta se aplicó de la siguiente manera: a la entrada del recinto ferial se solicitó la participación las personas que tuviesen 15 o más años de edad y que hubiesen consumido "alegría” al menos en una ocasión, de esa manera tendrían la experiencia que les permitiera responder el cuestionario empleado. Para motivarlos a responder el cuestionario, se les obsequió una "alegría”, además de explicarles que se trataba de un estudio con fines académicos.

Para medir los indicadores se usó una escala tipo Likert de siete puntos. La técnica para el análisis estadístico fue el modelo de ecuaciones estructurales. Las relaciones causales entre las variables endógenas y exógenas postuladas en las hipótesis se presentan en la Figura 1. Se empleó la matriz de varianza-covarianza de todos los indicadores usados en el modelo como datos de entrada, y el modelo se estimó con la función de máxima verosimilitud mediante el programa para analizar modelos de ecuaciones estructurales LISREL 8.80. El programa SPSS v.19.o se utilizó para el análisis descriptivo de los datos.

DOI: 10.22201 /enesl.20078064e.2018.16.62956

\section{Figura 1. Modelo estructural del valor percibido en el consumo de "alegría"}

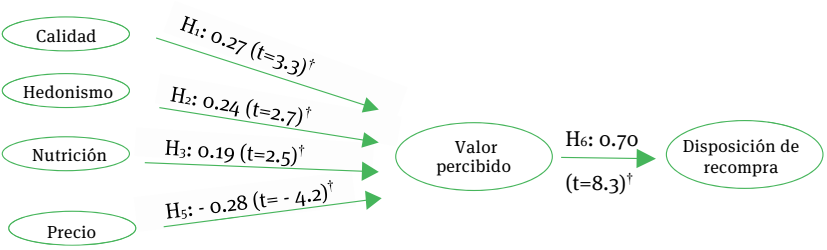

Fuente: elaboración propia con base en revisión de literatura.

\section{RESULTADOS}

Asistieron a la Feria 427 personas mayores de 15 años y se obtuvieron 370 cuestionarios autoadministrados por los asistentes, desechando 29 por tener datos incompletos. Por otra parte, se excluyó la variable salud porque $64 \%$ de los encuestados indicó no saber sobre las propiedades benéficas para la salud humana que tiene la "alegría” y que se mencionan en el cuestionario; sin embargo, del resto de los encuestados, $97 \%$ afirma que tiene esos beneficios en la salud. En tanto que $78 \%$ de los encuestados respondió a la pregunta sobre inocuidad, y de éstos, 98\% afirma que no causa algún daño a la salud.

Haciendo un desglose de la muestra se encontró que la cantidad de mujeres fue $6 \%$ mayor que la de hombres. Respecto a la edad encontramos que 2 de cada 10 entrevistados se encontraban entre 21 a 25 años; en el rango de menores de 30 años se encontró $43.9 \%$ de la muestra, $34.1 \%$ fueron menores de 50 años y $22 \%$ fueron personas mayores de 50 años. Respecto a la escolaridad los resultados fueron: primaria (17\%), secundaria $(13 \%)$, preparatoria $(22 \%)$, licenciatura $(36 \%)$, y posgrado (12.4\%). Se puede afirmar que los niveles educativos fueron altos, ya que aproximadamente la mitad de la muestra cuenta con estudios superiores.

Un aspecto importante para el consumo de un producto es el poder adquisitivo. Dentro de la encuesta se pidió al entrevistado que autoclasificara su condición socioeconómica y se encontró que $11.8 \%$ se ubicó como bajo, $11.4 \%$ como media-baja, $62.7 \%$ como media,12.2\% como media-alta y solamente $1.8 \%$ se manifestó como de condición socioeconómica alta. La mayor parte de los entrevistados se consideran en 
condición socioeconómica media y este aspecto es importante para adquirir el producto. Analizando en forma conjunta, frecuencia de compra y nivel socioeconómico se identificó que el promedio de compra de "alegría” en 12 meses fue de 16, 20.5, 24.9, 63.6, y 82 ocasiones para los encuestados con nivel socioeconómico bajo, medio bajo, medio, medio alto y alto, respectivamente.

Sobre el origen de los participantes, la gran mayoría (85.9\%) eran nativos del estado de Puebla y en menor frecuencia de otros estados como Tlaxcala (3.5\%), Morelos (2.6\%), Ciudad de México (2.4\%), Estado de México (2.1) y el resto de otras entidades como Oaxaca, Hidalgo, Chiapas, Veracruz, Guanajuato y Sinaloa.

La fiabilidad de escala de cada variable fue aceptable al tener un valor del alfa de Cronbach cercano o mayor al valor umbral de 0.7 recomendado por Hair et al. (2006). Sin embargo, tres indicadores de la variable calidad -apariencia, tamaño y envoltura- tuvieron un valor de fiabilidad individual igual o inferior a 0.53 por lo que fueron excluidos del modelo analizado.

El modelo propuesto estuvo identificado, al no presentar error alguno que evitara su estimación. El ajuste global del modelo fue aceptable, puesto que los valores de los tres tipos de medidas de ajuste -absoluto, incremental y de parsimonia- estuvieron en los rangos recomendados, tal como lo señala Hair et al. (2006), como se observa en el Cuadro 1. Esto indica que es fundada la aproximación del modelo al fenómeno bajo estudio y por tanto su poder de predicción.

\section{Cuadro 1. Medidas de bondad del ajuste del modelo estimado}

\begin{tabular}{llll}
\hline $\begin{array}{l}\text { Tipo } \\
\text { de medida }\end{array}$ & Medida & $\begin{array}{l}\text { Valor } \\
\text { estimado }\end{array}$ & $\begin{array}{l}\text { Valor } \\
\text { recomendado }\end{array}$ \\
\hline Absoluto: & Ji-cuadrada $(\mathrm{p}=0,00)$ & 109 & \\
& Grados de libertad & 43 & \\
& Índice de bondad de ajuste (GFI) & 0.95 & Máximo \\
& Residuo cuadrático medio (RMSR) & 0.026 & Mínimo \\
& Error de aproximación cuadrático medio (RMSEA) & 0.069 & $<0.08$ \\
Incremental: & Índice de bondad del ajuste incremental (AGFI) & 0.91 & $>0.9$ \\
& Índice Tucker-Lewis (TLI) o (NNFI) & 0.94 & $>0.9$ \\
& Índice de ajuste normado (NFI) & 0.94 & $>0.9$ \\
\hline Parsimonia: & Ji-cuadrada / Grados de libertad & 2.5 & $<3$
\end{tabular}

Fuente: elaboración propia a partir del análisis de resultados en LISREL.
El ajuste del modelo de medida fue aceptable, por lo siguiente: $a$ ) la fiabilidad individual de los indicadores fue aceptable al tener ponderaciones estimadas cercana o superiores al valor recomendado de 0.7 , y fueron estadísticamente significativas de acuerdo a los valores de t obtenidos (Hulland, 1999); b) la validez convergente fue aceptable al tener las variables una fiabilidad compuesta próxima o superior a 0.7 y una varianza extraída superior a 0.5 (Nunnally, 1978; Cuadro 2); c) la validez discriminante también fue aceptable debido a que la varianza extraída de cada variable es mayor al cuadrado de la correlación entre un constructo y todos los demás (Fornell y Larcker, 1981), y $d$ ) la validez de contenido se sustenta en la literatura revisada para seleccionar los indicadores de cada variable.

\section{Cuadro 2. Valores estimados de fiabilidad y validez para el modelo de medida}

\begin{tabular}{|c|c|c|c|c|c|c|}
\hline $\begin{array}{l}\text { Variables } \\
\text { e indicadores; }\end{array}$ & $\begin{array}{l}\text { Ponderación } \\
\text { estandarizada }\end{array}$ & Valor de $t$ & $\mathbf{R}^{2}$ & $\begin{array}{l}\text { Alfa de } \\
\text { Cronbach }\end{array}$ & $\begin{array}{l}\text { Fiabilidad } \\
\text { compuesta }\end{array}$ & $\begin{array}{l}\text { Varianza } \\
\text { extraída }\end{array}$ \\
\hline Calidad & & & & 0.72 & 0.72 & 0.56 \\
\hline Sabor & 0.75 & $12.3^{\dagger}$ & 0.57 & & & \\
\hline Textura & 0.74 & $12.1^{\dagger}$ & 0.55 & & & \\
\hline Hedonismo & & & & 0.75 & 0.75 & 0.6 \\
\hline Disfrute & 0.75 & $13.0^{\dagger}$ & 0.56 & & & \\
\hline $\begin{array}{l}\text { Sensación } \\
\text { de bienestar }\end{array}$ & 0.8 & $13.8^{\dagger}$ & 0.64 & & & \\
\hline Nutrición & & & & 0.72 & 0.72 & 0.57 \\
\hline $\begin{array}{l}\text { Fuente } \\
\text { de energía }\end{array}$ & 0.8 & $12.2^{\dagger}$ & 0.64 & & & \\
\hline Valor nutritivo & 0.71 & $11.2^{\dagger}$ & 0.5 & & & \\
\hline Precio & & & & 0.68 & 0.69 & 0.52 \\
\hline $\begin{array}{l}\text { Precio } \\
\text { económico }\end{array}$ & 0.71 & $9.0^{\dagger}$ & 0.5 & & & \\
\hline Precio relativo & 0.74 & $9.2^{\dagger}$ & 0.55 & & & \\
\hline Valor percibido & & & & 0.68 & 0.7 & 0.54 \\
\hline Compra valiosa & 0.8 & & 0.63 & & & \\
\hline $\begin{array}{l}\text { Valor } \\
\text { por dinero }\end{array}$ & 0.67 & $10.4^{\dagger}$ & 0.45 & & & \\
\hline Recompra & & & & 0.69 & 0.7 & 0.54 \\
\hline $\begin{array}{l}\text { Intención } \\
\text { de comprarla } \\
\text { nuevamente }\end{array}$ & 0.75 & & 0.56 & & & \\
\hline Recomendación & 0.72 & $8.7^{\dagger}$ & 0.53 & & & \\
\hline
\end{tabular}

Nota: $\uparrow \mathrm{p} \leq 0.01$

Fuente: elaboración propia a partir del análisis de resultados en LISREL.

El ajuste del modelo estructural fue aceptable al ser estadísticamente significativos todos los coeficientes 
estimados de las variables. El valor de $\mathrm{R}^{2}$ para las dos ecuaciones estructurales -valor percibido y disposición de recompra- fue 0.5 y 0.49 respectivamente.

\section{Discusión}

De acuerdo con Nicholson (2008) un bien normal es aquel cuya demanda aumenta (o permanece constante) a medida que el ingreso del individuo aumenta. Con base en dicha afirmación, resultados del presente trabajo proporcionan indicios de que la "alegría" es un bien normal: encuestados con mayor nivel socioeconómico adquieren con mayor frecuencia la "alegría".

Los resultados obtenidos mediante la estimación del modelo propuesto y la evaluación de la calidad de los tipos de ajuste - global, medida y estructural- soportan la afirmación de que existe una relación significativa y positiva entre las dimensiones de beneficios analizadas calidad, hedonismo y nutrición - y el valor percibido por el cliente, por lo cual se aceptan las tres primeras hipótesis, H1: la calidad de la "alegría” contribuye a aumentar el valor que el consumidor percibe en este alimento; $\mathrm{H2}$ : las características hedónicas de la "alegría” contribuyen a aumentar el valor que el consumidor percibe en este alimento, y H3: las propiedades nutritivas de la "alegría” contribuyen a aumentar el valor que el consumidor percibe en este alimento. La cuarta dimensión de beneficios, que es la de salud, no se analizó por datos insuficientes, por lo cual no es aceptada. Con respecto al costo asociado al valor percibido, se puede afirmar que existe una relación significativa y negativa entre el costo monetario y el valor percibido por el cliente, por lo tanto se acepta la quinta hipótesis (H5: el precio de la "alegría" contribuye a disminuir el valor que el consumidor percibe en este alimento). En cuanto a la lealtad del consumidor, se puede afirmar que existe una relación significativa y positiva entre el valor percibido por el cliente y la disposición de recompra, lo que permite aceptar la sexta hipótesis (H6: el valor que el consumidor percibe en la "alegría” influye positivamente en su disposición de recompra). Estos resultados se presentan en la Figura 2.

DOI: $10.22201 /$ enesl .20078064 e.2018.16.62956
Figura 2. Modelo estructural contrastado

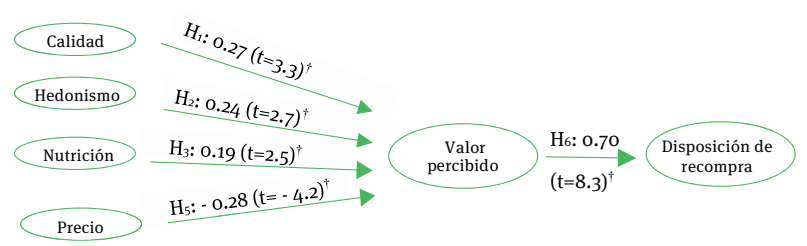

Fuente: elaboración propia a partir del análisis de resultados en LISREL.

El precio fue la dimensión con mayor impacto, aunque en forma negativa, en la conformación del valor que el consumidor percibe de la "alegría”. Esto significa que el consumidor encuestado tiene muy en cuenta el desembolso que hace al comprar una "alegría". Un factor que pudo influir para que se diera esta apreciación es la característica socioeconómica de los encuestados, puesto que $86 \%$ de ellos tenía uno de los tres niveles inferiores de la escala utilizada. Este resultado está en concordancia con el hecho de que, habitualmente para la mayoría de los clientes, es el sacrificio más importante que hacen al comprar un producto o servicio, y múltiples autores afirman que es uno de los más sobresalientes componentes que determinan el valor percibido (Morar, 2013).

La calidad, con un coeficiente estimado muy cercano al del precio, fue la segunda dimensión que tuvo mayor relevancia sobre el valor percibido en la "alegría”, lo cual está en concordancia con lo que sucede en casi todo tipo de productos, incluyendo los alimentos: la calidad es uno de los elementos importantes en la percepción y decisión de compra del consumidor, tal y como ha sido corroborado en diferentes investigaciones (Rotaris y Danielis, 2011; Shaharudin, Wan y Elias 2011).

El componente hedónico de la "alegría”, fue la tercera dimensión con mayor contribución para conformar el valor percibido, siendo un resultado razonable puesto que el alimento es una de las principales fuentes de experiencias hedónicas en la vida humana (Luomala, Laaksonen y Leipämaa, 2004). Este hecho refuerza la propuesta de concebir la calidad y el hedonismo como dimensiones independientes del valor percibido en el consumo de alimentos y bebidas, y es una evidencia adicional a lo reportado por estudios previos, en donde también se identificó que la calidad, el precio y lo hedónico son las dimensiones de valor más importantes en esa categoría 
de productos (Aragón et al., 2013).

La nutrición fue la dimensión, de las que se estudiaron, que menos aportó en la creación de valor que percibe el consumidor; sin embargo, es relevante que los consumidores aprecian sus propiedades nutritivas, situación que tiene mayor importancia al tomar en cuenta que la "alegría” se oferta, casi en la totalidad de los casos, sin información alguna sobre su aporte nutritivo. Por otra parte, este resultado está en concordancia con la teoría del comer hedónico (Stroebe, Papies y Aarts, 2008), en donde se establece que la anticipación del placer que se puede derivar de los alimentos es un determinante más poderoso que sus propiedades nutritivas, en la decisión de los consumidores.

La dimensión salud no se analizó porque $64 \%$ de los encuestados indicó no saber sobre las propiedades benéficas para la salud humana que tiene la "alegría”. Este hecho implica la necesidad de comunicar de manera eficaz los beneficios a la salud que puede aportar este tipo de alimento a fin de potenciar su consumo; sin embargo, como lo afirman Mai y Hoffmann (2015), una campaña de información hacia lo saludable no es suficiente, requiriéndose cambiar el paradigma que afirma "lo saludable requiere compromiso (educación y conocimiento)" por el que enuncia "lo saludable es sabroso".

Por último, se puede mencionar que los resultados obtenidos confirman que la teoría del VPC es un medio eficaz para estudiar el comportamiento del consumidor y explicar su lealtad hacia los productos que adquiere.

\section{CONCLUSIONES}

Con respecto a la valoración que los encuestados hicieron sobre los atributos de la "alegría", se concluye que el precio y la calidad, al igual que en la mayoría de las categorías de productos, son los componentes más importantes en la conformación del VPC, en tanto que sus características hedónicas están muy cerca de equipararse con aquellas que más contribuyeron en crear valor para los consumidores encuestados, por lo que el placer que genera tiene preeminencia en sus preferencias sobre otras dimensiones como lo es la nutrición, siendo ésta la que menos contribuyó a la creación del valor percibido; sin embargo, su aportación es significativa, por lo que puede esperarse que los consumidores encuestados prefieran, entre dos alimentos que produzcan el mismo placer, aquel que perciban con mayor valor nutricional.

En cuanto a las propiedades benéficas para la salud que tiene la "alegría”, éstas son desconocidas por la mayoría de los encuestados, por lo que es primordial su difusión, debiendo ser de manera convincente, puesto que son características que para el consumidor son muy difíciles de verificar. Por otra parte, se identificó un avance importante en la dimensión salud con respecto a la inocuidad de la "alegría", puesto que $76 \%$ de los encuestados reconoce que la "alegría" es un alimento inocuo para la salud, lo que hace suponer que si se hubiese podido incluir esta variable en el modelo de análisis, seguramente hubiese aumentado significativamente la proporción de variación explicada en las respuestas de los encuestados.

Con este análisis dimensional de las propiedades de la "alegría", como un caso específico de los alimentos con alto valor nutritivo y saludable, se hace una aportación para continuar avanzando en el desarrollo del conocimiento sistematizado del valor percibido por el consumidor.

Por otra parte, con base en el conocimiento generado sobre las preferencias del consumidor y ante la obesidad que afecta a la mayoría de los mexicanos, es recomendable promover el consumo de alimentos sabrosos, nutritivos, saludables, con bajo contenido de grasas, preferentemente que sean productos locales y con un precio accesible para los sectores de la sociedad con menores ingresos, tal como lo es la "alegría".

Con el propósito de reforzar aquellas estrategias que busquen potenciar la demanda de alimentos nutritivos y saludables, se sugiere realizar estudios complementarios para identificar las principales barreras que limitan su consumo. 


\section{REFERENCIAS}

Aragón, C., Montero, M., Araque, R., y Gutiérrez, L. (2013). Evaluación del valor percibido en el consumo de café con atributos éticos. Agrociencia, 47(2), 195-207.

Astiasarán, I. y Martínez J. (2000). Alimentos: composición y propiedades. España: McGraw-Hill Interamericana.

Ayala, A., Escobedo, D., Cortés, L., y Espitia, E. (2012). El cultivo de amaranto en México, descripción de la cadena, implicaciones y retos. En: E. EspitiaRangel (Ed.), Amaranto: Ciencia y Tecnología (pp. 315-330). México: Instituto Nacional de Investigaciones Forestales, Agrícolas y Pecuarias.

Ayala, A., Espitia, E., Rivas, P., Martínez, G., y Almaguer, G. (2016). Análisis de la cadena del valor de amaranto en México. Agricultura, Sociedad y Desarrollo, 13(1), 87-104.

Ayyaz, S., Badar, H., y Ghafoor, A. (2011). Level and determinants of consumers' perception of packed milk in Pakistan. Journal of Business \& Economics, 3(1), 60-76.

Beeler, J., McCutcheon, J., Cao, Z., Murakami, M., Alexander, E., Roitman, M., y Zhuang, X. (2012). Taste uncoupled from nutrition fails to sustain the reinforcing properties of food. European Journal of Neuroscience, 36(4), 2533-2546.

Berry, L., Seiders, K., y Grewal, D. (2002). Understanding Service Convenience. Journal of Marketing, 66(3), 1-17.

Çağlarırmak, N., y Zeki, H. (2013). An investigation of nutritional values of dried vegetables. GIDA / The Journal of FOOD, 38(6), 327-333.

Castillo, R. (2013). El hambre en México. Estudios: Filosofía, Historia, Letras, (107), 71-111.

Chaput, J., Klingenberg, L., Astrup, A., y Sjodin, A. M. (2011). Modern sedentary activities promote overconsumption of food in our current obesogenic environment. Obesity Reviews, 12(5), 12-20.

Chávez, A., Ledesma, J., Mendoza, E., Calvo, M., Castro, M., Ávila, A. ... (2013). Tablas de uso práctico de los alimentos de mayor consumo. México: McGraw-Hill Interamericana.

Chen, Z., y Dubinsky, A. (2003). A conceptual model of perceived customer value in e-commerce: a pre- liminary investigation. Psychology \& Marketing, 20(4), 323-347.

Consejo Nacional de Evaluación de la Política de Desarrollo Social [Coneval]. (2015). Medición de la pobreza en México y en las Entidades Federativas 2014. México: Coneval.

Dutta, K., y Singh, S. (2013). Healthy food behavior and celebrity endorsement for healthy food brands: a study of indian housewives. IUP Journal of Brand Management, 10(3), 33-46.

Escobedo, D., Ayala, A., y Campos, L. (2012). Formas de consumo del amaranto en México. En: E. EspitiaRangel (Ed.), Amaranto: Ciencia y Tecnología (pp. 341-354). México: Instituto Nacional de Investigaciones Forestales, Agrícolas y Pecuarias.

Espitia, E., Mapes, C., Escobedo, D., De la O, M., Rivas, P., Martínez, G...(2010). Conservación y uso de los recursos genéticos de Amaranto en México. México: Sinarefi-INIFAP-UNAM.

Fornell, C., y Larcker, D. (1981). Evaluating Structural Equation Models with Unobservable Variables and Measurement Error. Journal of Marketing Research, 18, 39-50.

Gallarza, M., Gil, I., y Holbrook, M. (2011). The value of value: Further excursions on the meaning and role of customer value. Journal of Consumer Behaviour, 10(4), 179-191.

Grewal, D., Monroe, K., y Krishnan, R. (1998). The effects of price-comparison advertising on buyers' perceptions of acquisition value, transaction value, and behavioral intentions. Journal of Marketing, 62(2), 46-59.

Hair, J. F., Black, W. C., Babin, B. J., Anderson, R. E., y Tatham, R. L. (2006). Multivariate data analysis. New Jersey: Prentice Hall.

Holbrook, M. (1999). Consumer value: A framework for analysis and research. London: Routledge.

Huerta, J., Maldonado, E., y Barba, A. (2012). Amaranto: propiedades benéficas para la salud. En: E. Espitia-Rangel (Ed.), Amaranto: Ciencia y Tecnología (pp. 303-312). México: Instituto Nacional de Investigaciones Forestales, Agrícolas y Pecuarias.

Hulland, J. (1999). Use of partial least squares (PLS) in strategic management research: a review of four 
recent studies. Strategic Management Journal, 20(2), 195-204.

Jezewska, M., y Krolak, M. (2015). Do consumers' attitudes towards food technologies and motives of food choice influence willingness to eat cereal products fortified with fibre? Polish Journal of Food and Nutrition Sciences, 65(4), 281-291.

Kaufer, M., Pérez, A., y Arroyo, P. (2015). Nutriología médica. México: Editorial Médica Panamericana.

Krugman, P., y Wells R. (2006). Introducción a la Economía. Microeconomía. España: Editorial Reverté, S.A.

Lehtola, K., Luomala, H., Kauppinen, H., Kupiainen, T., y Tuuri, H. (2008). Consumers' experience of food products: effects of value activation and price cues. Journal of Customer Behaviour, 7(1), 19-29.

Luomala, H., Laaksonen P., y Leipämaa, H. (2004). How Do Consumers Solve Value Conflicts in Food Choices? An Empirical Description and Points for Theory-building. Advances in Consumer Research, 31(1), 564-570.

Mai, R., y Hoffmann, S. (2015). How to Combat the Unhealthy $=$ Tasty Intuition The Influencing Role of Health Consciousness. Journal of Public Policy \& Marketing, 34(1), 63-83.

Morar, D. (2013). An overview of the consumer value literature - perceived value, desired value. International Conference "Marketing - from information to decision", 6, 169-186.

Morán, S., Cortés, L., Espitia, E., y Sangerman, D. (2012). Tzoalli, de cuerpo de dioses a alegría de mortales. En: E. Espitia-Rangel (Ed.), Amaranto: Ciencia y Tecnología (pp.15-27). México: Instituto Nacional de Investigaciones Forestales, Agrícolas y Pecuarias.

Nasir, V., y Karakaya, F. (2014). Underlying motivations of organic food purchase intentions. Agribusiness, 30(3), 290-308.

Nicholson, W. (2008). Teoría microeconómica. Principios básicos y aplicaciones ( $9^{a}$ edición). México: Cengage Learning Editores, S.A. de C.V.

Nunnally, J. (1978). Psychometric Theory. New York: McGraw Hill.

Pacheco, R., Mota, L., y Luna, N. (2017). Estado Nutricio, Nivel de Hemoglobina y Calidad de la Dieta en Niños Menores de 5 Años de Zonas Rurales de
Ejutla de Crespo y Coatecas Altas, Oaxaca. Salud y Administración, 12(4), 3 - 14.

Parasuraman, A., y Grewal, D. (2000). The impact of technology on the quality-value-loyalty chain: a research agenda. Journal of the Academy of Marketing Science, 28, 168-174.

Petrick, J. (2002). Development of a multi-dimensional scale for measuring the perceived value of a service. Journal of Leisure Research, 34(2), 119-134.

Pérez, S., y Romero, G. (2018). Imagen corporal y representaciones alimentarias en mujeres de trece comunidades rurales mexicanas. Población y Salud en Mesoamérica, 15(2). Recuperado de http:// dx.doi.org/10.15517/psm.v15i2.31634

Richards, T., Patterson, P. and Hamilton, S. (2007). Fast food, addiction, and market power. Journal of Agricultural \& Resource Economics, 32(3), 425-447.

Rotaris, L., y Danielis, R. (2011). Willingness to Pay for Fair Trade Coffee: A Conjoint Analysis Experiment with Italian Consumers. Journal of Agricultural \& Food Industrial Organization, 9(1), 1-20.

Sánchez, J., Argumedo, A., Álvarez, J., Méndez, J., y Ortiz, B. (2015). Conocimiento tradicional en prácticas agrícolas en el sistema del cultivo de amaranto en Tochimilco, Puebla. Agricultura, Sociedad y Desarrollo, 12(2), 237-254.

Sánchez, R., Iniesta, M., y Holbrook, M. (2009). The conceptualisation and measurement of consumer value in services. International Journal of Market Research, 51(1), 93-113.

Secretaría de Agricultura, Ganadería, Desarrollo Rural, Pesca y Alimentación [Sagarpa]. (2013). Servicio de Información Agroalimentaria y Pesquera. México. Recuperado de http://infosiap.siap.gob.mx/ aagricola_siap_gb/ientidad/index.jsp

Secretaría de Agricultura, Ganadería, Desarrollo Rural, Pesca y Alimentación [Sagarpa]. (2016a). Servicio de Información Agroalimentaria y Pesquera. México. Recuperado de http://infosiap.siap.gob.mx/ aagricola_siap_gb/ientidad/index.jsp

Secretaría de Agricultura, Ganadería, Desarrollo Rural, Pesca y Alimentación [Sagarpa]. (2016b). Servicio de Información Agroalimentaria y Pesquera. Amaranto. México. Recuperado de https://www. gob.mx/cms/uploads/attachment/file/166367/ amaranto_monograf_a.pdf 
Shaharudin, M., Wan, M., y Elias, S. (2011). Food Quality Attributes among Malaysia's Fast Food Customer. International Business \& Management, 2(1), 198208.

Smith, N. (1999). Ethics and the typology of consumer value. En: M. Holbrook (Ed.), Consumer value. A framework for analysis and research (pp. 43-62). Londres: Routledge.

Stroebe, W., Papies, E., y Aarts, H. (2008). From homeostatic to hedonic theories of eating: self-regulatory failure in food-rich environments. Applied Psychology: An International Review, 57, 172-193.

Sweeney, J., Soutar, G., y Johnson, L. (1999). The role of perceived risk in the quality-value relationship: a study in a retail environment. Journal of Retailing, 75(1), 77-105.

United Nations International Children's Emergency Fund. (s.f.). Salud y nutrición: el doble reto de la malnutrición y la obesidad. Recuperado de http:// www.unicef.org/mexico/spanish/17047.htm

Wenben, A. (1995). Consumer Values, Product Benefits and Customer Value: A Consumption Behavior Approach. Advances in Consumer Research, 22(1), 381-388. 


\section{ANEXO 1. Cuestionario}

\section{COLEGIO DE POSTGRADUADOS EN CIENCIAS AGRICOLAS}

Trabajo de Investigación Doctoral en Mercadotecnia, por Carlos Aragón Gutiérrez Este cuestionario tiene el propósito de conocer las percepciones que el consumidor tiene del dulce "alegría"

Por favor, marca con una X una de las siete opciones, en cada oración, que mejor refleje tu percepción del dulce “alegría”

Tiene un sabor:

Comerla es:

La "alegría" me da:

Reduce la presión

arterial alta:

El precio de la "alegría" es:

Comprar una "alegría" es:

Con gusto la compraré

nuevamente:

Tiene una dureza

o consistencia:

Comerla me

hace sentir:

La "alegría" también

es un buen alimento:

Tiene efectos preventivos contra el cáncer:

La "alegría" es:

La "alegría" vale lo

que pago:

La recomendaré

a otras personas:

Tiene una apariencia:

Compro la "alegría" por:

Tiene proteínas,

vitaminas y minerales:

Estimula el sistema

inmunológico para evitar

enfermedades infecciosas:

En comparación con otras

golosinas, su precio es:

La "alegría" es una

golosina valiosa:

Tiene un tamaño:

No causa algún daño

a la salud:

\begin{tabular}{|c|c|c|c|c|c|c|}
\hline $\begin{array}{l}\text { muy } \\
\text { malo }\end{array}$ & malo & $\begin{array}{l}\text { un poco } \\
\text { malo }\end{array}$ & indiferente & $\begin{array}{l}\text { un poco } \\
\text { bueno }\end{array}$ & bueno & $\begin{array}{c}\text { muy } \\
\text { bueno }\end{array}$ \\
\hline $\begin{array}{c}\text { muy } \\
\text { desagradable }\end{array}$ & desagradable & \begin{tabular}{|c|} 
un poco \\
desagradable
\end{tabular} & indiferente & $\begin{array}{l}\text { un poco } \\
\text { agradable }\end{array}$ & agradable & $\begin{array}{c}\text { muy } \\
\text { agradable }\end{array}$ \\
\hline
\end{tabular}

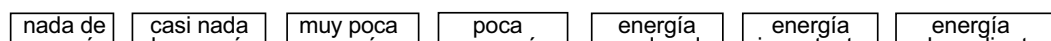
$\begin{gathered}\text { nada de } \\ \text { energía }\end{gathered}$
de energía $\begin{gathered}\begin{array}{c}\text { muy poca } \\ \text { energía }\end{array} \\ \text { energía }\end{gathered} \begin{gathered}\text { pergía } \\ \text { moderada }\end{gathered} \begin{gathered}\text { energía } \\ \text { importante }\end{gathered} \begin{gathered}\text { energía } \\ \text { sobresaliente }\end{gathered}$

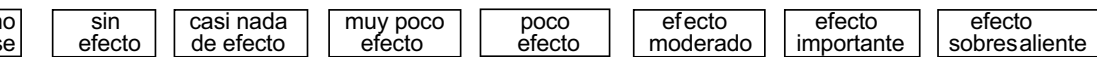
\begin{tabular}{|c|c|c|c|c|c|}
\hline $\begin{array}{c}\text { muy } \\
\text { inaceptable }\end{array}$ & un poco \\
inaceptable & $\begin{array}{l}\text { muy poco } \\
\text { aceptable }\end{array}$ inaceptable \\
indiferente
\end{tabular}

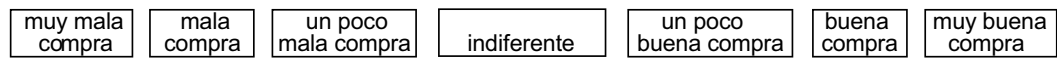

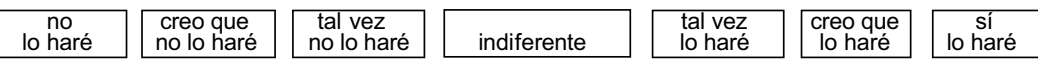
$\begin{gathered}\text { muy } \\
\text { mala }\end{gathered}$ mala \begin{tabular}{c|c|c|c|} 
un poco \\
mala
\end{tabular} indiferente
un poco
buena buena $\begin{gathered}\text { muy } \\
\text { buena }\end{gathered}$

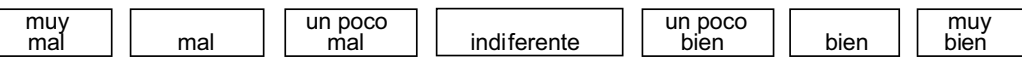

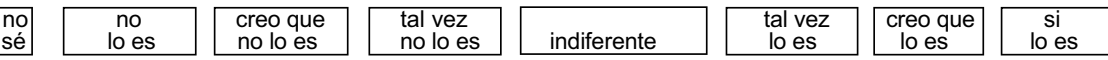

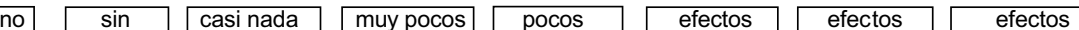

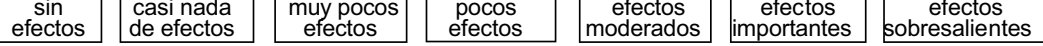

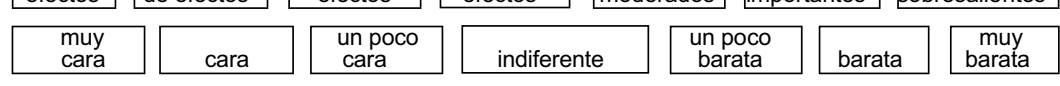

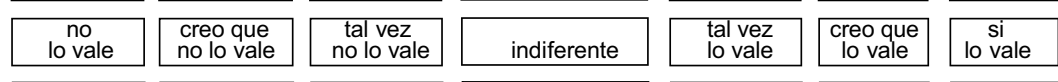

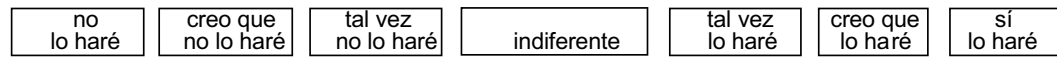

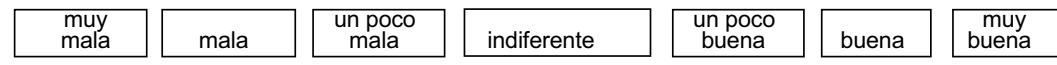
gran necesidad necesidad poca necesidad necesidad de un poco un gran

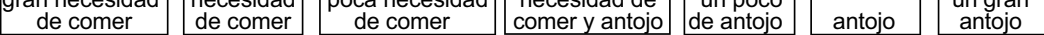

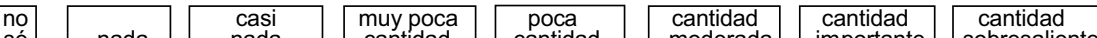

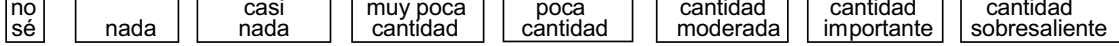

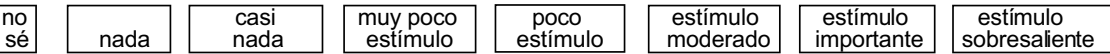

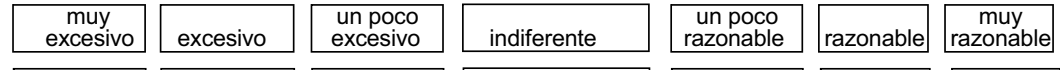

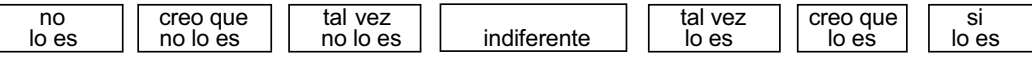

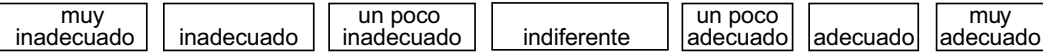

Deseo la compren más personas:

Tiene una envoltura:

\begin{tabular}{|c|c|c|c|c|c|c|c|c|}
\hline $\begin{array}{r}\text { causa } \\
\text { daño }\end{array}$ & $\begin{array}{l}\text { creo que } \\
\text { causa daño }\end{array}$ & \begin{tabular}{|c|} 
tal vez \\
causa daño
\end{tabular} & indiferente & $\begin{array}{l}\text { tal v } \\
\text { cau }\end{array}$ & âno & $\begin{array}{l}\text { creo } \\
\text { caus }\end{array}$ & $\begin{array}{l}\text { que no } \\
\text { a daño }\end{array}$ & $\begin{array}{c}\text { no causa } \\
\text { daño }\end{array}$ \\
\hline $\begin{array}{c}\text { no } \\
\text { es así }\end{array}$ & $\begin{array}{l}\text { creo que } \\
\text { no es así }\end{array}$ & $\begin{array}{c}\text { tal vez } \\
\text { no es así }\end{array}$ & \multicolumn{2}{|c|}{ indiferente } & \multicolumn{2}{|c|}{$\begin{array}{l}\text { tal vez } \\
\text { es así }\end{array}$} & $\begin{array}{l}\text { creo que } \\
\text { es así }\end{array}$ & $\begin{array}{l}\text { es } \\
\text { así }\end{array}$ \\
\hline $\begin{array}{c}\text { muy } \\
\text { inadecuada }\end{array}$ & inadecua & $\begin{array}{r}\text { un po } \\
\text { inadecu }\end{array}$ & lada & rente & & uada & adecuada & $\begin{array}{c}\text { muy } \\
\text { adecuada }\end{array}$ \\
\hline
\end{tabular}

En cuántas ocasiones ha comprado "alegrías" en los 12 meses recientes:

DATOS DEL ENCUESTADO Estado

Nivel máximo de estudios: primaria__ secundaria__ preparatoria_ licenciatura__ postgrado__

Condición socioeconómica: baja__ media baja__ media__ media alta__ alta__ 


\section{NOTAS DE AUTOR}

a Doctor en Planeación y Dirección de la Empresa en el Entorno Social. Profesor Investigador Asociado; Colegio de Postgraduados, Campus Puebla. Líneas de investigación: Comercialización de productos agrícolas con atributos éticos. Correo electrónico: carlos.aragon@colpos.mx.

b Doctor en Estudios Latinoamericanos. Colegio de Postgraduados, Campus Puebla; Profesor Investigador Titular. Líneas de investigación: Desarrollo Rural. Correo electrónico: bramirez@colpos.mx. *Autor de correspondencia

c Doctora en Administración de Empresas. Universidad Loyola Andalucía; Directora Departamento Gestión Empresarial. Líneas de investigación: Marketing orientado a la transformación social. Correo electrónico: jmontero@uloyola.es.

d Doctor en Administración de Empresas. Universidad Loyola Andalucía; Profesor Titular. Líneas de investigación: Marketing orientado a la transformación social. Correo electrónico: raraque@uloyola. es.

e Doctor en Administración de Empresas. Universidad Loyola Andalucía; Profesor Titular. Líneas de investigación: Marketing orientado a la transformación social. Correo electrónico: jjperez@uloyola.es 